\title{
STATISTICAL ANALYSIS OF SIMULATION OUTPUT DATA: THE PRACTICAL STATE OF THE ART
}

\author{
Averill M. Law \\ Averill M. Law \& Associates \\ 4729 East Sunrise Drive, \#462 \\ Tucson, AZ 85718, USA
}

\begin{abstract}
One of the most important but neglected aspects of a simulation study is the proper design and analysis of simulation experiments. In this tutorial we give a state-of-the-art presentation of what the practitioner really needs to know to be successful. We will discuss how to choose the simulation run length, the warmup-period duration (if any), and the required number of model replications (each using different random numbers). The talk concludes with a discussion of three critical pitfalls in simulation output-data analysis.
\end{abstract}

\section{INTRODUCTION}

In many "simulation studies" a great amount of time and money is spent on model development and "programming," but little effort is made to analyze the simulation output data appropriately. As a matter of fact, a very common mode of operation is to make a single simulation run of somewhat arbitrary length and then to treat the resulting simulation estimates as the "true" model characteristics. Since random samples from probability distributions are typically used to drive a simulation model through time, these estimates are just particular realizations of random variables that may have large variances. As a result, these estimates could, in a particular simulation run, differ greatly from the corresponding true characteristics for the model. The net effect is, of course, that there could be a significant probability of making erroneous inferences about the system under study.

We now describe more precisely the random nature of simulation output. Let $Y_{1}, Y_{2}, \ldots$ be an output stochastic process [see, for example, section 4.3 in Law (2007)] from a single simulation run. For example, $Y_{i}$ might be the delay in queue for the $i$ th job to arrive at a single-server queueing system. Alternatively, $Y_{i}$ might be the total cost of operating an inventory system in the $i$ th month. The $Y_{i}{ }^{\prime} s$ are random variables that will not, in general, be independent or identically distributed (IID). Thus, many of the formulas from classical statistics (see Section 2) will not be directly applicable to the analysis of simulation output data.

Example 1. For the queueing system mentioned above, the delays in queue will not be independent, since a large delay for one customer waiting in queue will tend to be followed by a large delay for the next customer waiting in queue. Suppose that the simulation is started at time zero with no customers in the system, as is usually the case. Then the delays in queue at the beginning of the simulation will tend to be smaller than later delays and, thus, the delays are not identically distributed.

Let $y_{11}, y_{12}, \ldots, y_{1 m}$ be a realization of the random variables $Y_{1}, Y_{2}, \ldots, Y_{m}$ resulting from running the simulation with a particular set of random numbers $u_{11}, u_{12}, \ldots$. If we run the simulation with a different 
set of random numbers $u_{21}, u_{22}, \ldots$, then we will obtain a different realization $y_{21}, y_{22}, \ldots, y_{2 m}$ of the random variables $Y_{1}, Y_{2}, \ldots, Y_{m}$. (The two realizations are not the same since the different random numbers used in the two runs produce different samples from the input probability distributions.) In general, suppose that we make $n$ independent replications (runs) of the simulation (i.e., different random numbers are used for each replication, each replication uses the same initial conditions, and the statistical counters for the simulation are reset at the beginning of each replication) each of length $m$, resulting in the observations:

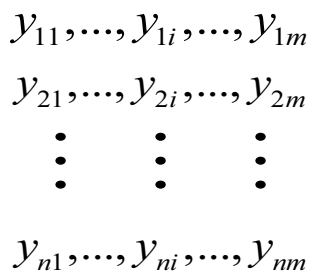

The observations from a particular replication (row) are clearly not IID. However, note that $y_{1 i}, y_{2 i}, \ldots, y_{n i}$ (from the $i$ th column) are IID observations of the random variable $Y_{i}$, for $i=1,2, \ldots, m$. More generally, each entire replication is independent of any other replication, and each replication's observations have the same (joint) distribution. This independence across runs is the key to relatively simple output-data analysis that is discussed in later sections of this paper. Then, roughly speaking, the goal of output-data analysis is to use the observations $y_{j i}(i=1,2, \ldots, m ; j=1,2, \ldots, n)$ to draw inferences about characteristics of the random variables $Y_{1}, Y_{2}, \ldots, Y_{m}$.

Example 2. Consider a bank with five tellers and one queue, which opens its doors at 9 A.M., closes its doors at 5 P.M., but stays open until all customers in the bank at 5 P.M. have been served. Assume that customers arrive with IID exponential interarrival times with mean 1 minute, that service times are IID exponential random variables with mean 4 minutes, and that customers are served in a first-in, first-out (FIFO) manner. Table 1 shows two typical output statistics from 5 independent replications of the bank, assuming that no customers are present initially. Note that results from different replications can be quite different. Thus, one run clearly does not produce the "answers."

Table 1: Results for 5 Independent Replications of the Bank Model

\begin{tabular}{|c|c|c|}
\hline Replication & $\begin{array}{c}\text { Average delay } \\
\text { in queue }\end{array}$ & $\begin{array}{c}\text { Average number } \\
\text { in queue }\end{array}$ \\
\hline 1 & 1.53 & 1.52 \\
\hline 2 & 1.66 & 1.62 \\
\hline 3 & 1.24 & 1.23 \\
\hline 4 & 2.34 & 2.34 \\
\hline 5 & 2.86 & 2.83 \\
\hline
\end{tabular}

Our goal in this paper is to discuss methods for statistical analysis of simulation output data and to present the material with a practical focus. Section 2 of this paper reviews formulas from classical statistics based on IID data, which we will find useful later in this paper. In Section 3, we discuss the two main types of simulations with regard to output-data analysis, namely, terminating and non-terminating. Statistical methods for analyzing each type are given in Sections 4 and 5, respectively. Finally, we give a summary of this tutorial and three fundamental pitfalls in output-data analysis in Section 6. 
Portions of this paper are based on Chapters 4 and 9 of Law (2007). Other references on output-data analysis are Alexopoulos (2007), Banks et al. (2010), and Nakayama (2008).

\section{REVIEW OF CLASSICAL STATISTICS}

Suppose that $X_{1}, X_{2}, \cdots, X_{n}$ are IID random variables with population mean and variance $\mu$ and $\sigma^{2}$, respectively. Then unbiased point estimators for $\mu$ and $\sigma^{2}$ are given by

$$
\bar{X}(n)=\frac{\sum_{i=1}^{n} X_{i}}{n}
$$

and

$$
S^{2}(n)=\frac{\sum_{i=1}^{n}\left[X_{i}-\bar{X}(n)\right]^{2}}{n-1}
$$

Furthermore, an approximate $100(1-\alpha)$ percent $(0<\alpha<1)$ confidence interval for $\mu$ is given by

$$
\bar{X}(n) \pm t_{n-1,1-\alpha / 2} \sqrt{S^{2}(n) / n}
$$

where $t_{n-1,1-\alpha / 2}$ is the upper $1-\alpha / 2$ critical point for a $t$ distribution with $n-1$ degrees of freedom. If the sample size $n$ is "sufficiently large," then the confidence interval given by Expression (3) will have a coverage probability arbitrarily close to $1-\alpha$. Alternatively, if the $X_{i}{ }^{\prime} s$ are normally distributed, then the coverage probability will be exactly $1-\alpha$. In practice, if the distribution of the $X_{i}{ }^{\prime} s$ is reasonably symmetric, then the coverage probability will be close to $1-\alpha$ [see Law (2007, pp. 232-236)]. If we in-

crease the sample size from $n$ to $4 n$, then the half-length of the confidence interval, $t_{n-1,1-\alpha / 2} \sqrt{S^{2}(n) / n}$, will decrease by a factor of approximately 2 , since there is an $n$ in the denominator under the square-root sign.

As stated above, the $Y_{i}{ }^{\prime} s$ from one simulation run are not IID and, thus, Expressions (1), (2), and (3) are not directly applicable to their analysis. However, if we take comparable output statistics from different independent replications of a simulation model, then these observations are IID and the three expressions are applicable.

Example 3. For the bank simulation of Example 2, the five average delays in queue from column 2 of Table 1 are IID and, thus, Expressions (1), (2), and (3) could legitimately be used for their analysis.

\section{TYPES OF SIMULATIONS WITH REGARD TO OUTPUT ANALYSIS}

The options available for designing and analyzing simulation experiments depend on whether the simulation of interest is terminating or non-terminating, which depends on whether there is an obvious way for determining the simulation run length.

A terminating simulation is one for which there is a "natural" event $E$ that specifies the length of each run (replication). Since different runs use independent random numbers and the same initialization rule, this implies that comparable random variables are IID. The event $E$ often occurs at a time point that has one of the following properties: 
- The system is "cleaned out"

- Beyond which no useful information is obtained

- Specified by management.

The event $E$ is specified before any runs are made, and the time of occurrence of $E$ for a particular run may be a random variable. Since the initial conditions for a terminating simulation generally affect the desired measures of performance, these conditions should be representative of those for the actual system.

Example 4. A retail/commercial establishment (e.g., a bank) closes each evening. If the establishment is open from 9 to 5 , the objective of a simulation might be to estimate some measure of the quality of customer service over the period beginning at 9 A.M. and ending when the last customer who entered before the doors closed at 5 P.M. has been served. In this case, $E=\{8$ hours of simulated time have elapsed and the system is empty\}, and the initial conditions for the simulation should be representative of those for the bank at 9 A.M.

Example 5. Consider a military ground confrontation between a blue force and a red force. Relative to some initial force strengths, the goal of a simulation might be to determine the (final) force strengths when the battle ends. In this case, $E=$ \{either the blue force or the red force has "won" the battle\}. An example of a condition that would end the battle is one side losing 30 percent of its force, since this side would no longer be considered viable. The choice of initial conditions for the simulation, e.g., the number of troops and tanks for each force, is generally not a problem here, since they are specified by the military scenario under consideration.

A non-terminating simulation is one for which there is no natural event $E$ to specify the length of a run. This often occurs when we are designing a new system or modifying an existing system, and we are interested in the behavior of the system in the long run when it is operating "normally." Unfortunately, "in the long run" doesn't naturally translate into a terminating event $E$.

Consider the output stochastic process $Y_{1}, Y_{2}, \ldots$ for a simulation model. Let $F_{i}(y \mid I)=P\left(Y_{i} \leq y \mid I\right)$ for $i=1,2, \ldots$, where $y$ is a real number and $I$ represents the initial conditions used to start the simulation at time 0 . [The conditional probability $P\left(Y_{i} \leq y \mid I\right)$ is the probability that the event $\left\{Y_{i} \leq y\right\}$ occurs given the initial conditions $I$.] For a manufacturing system, $I$ might specify the number of jobs present, and whether each machine is busy or idle, at time 0 . We call $F_{i}(y \mid I)$ the transient distribution of the output process at (discrete) time $i$ for initial conditions $I$. Note that $F_{i}(y \mid I)$ will, in general, be different for each value of $i$ and each set of initial conditions $I$. For fixed $y$ and $I$, the probabilities $F_{1}(y \mid I), F_{2}(y \mid I), \ldots$ are just a sequence of numbers. If $F_{i}(y \mid I) \rightarrow F(y)$ as $i \rightarrow \infty$ for all $y$ and any initial conditions $I$, then $F(y)$ is called the steady-state distribution of the output process $Y_{1}, Y_{2}, \ldots$. Note that the steady-state distribution $F(y)$ does not depend on the initial conditions $I$.

A measure of performance for a non-terminating simulation is said to be a steady-state parameter if it is a characteristic of the steady-state distribution of some output stochastic process $Y_{1}, Y_{2}, \ldots$. If the random variable $Y$ has the steady-state distribution, then we are typically interested in estimating the steadystate mean $v=E(Y)$.

Example 6. Consider a company that is going to build a new manufacturing system and would like to determine the long-run (steady-state) mean hourly throughput of their system after it has been running long enough for workers to know their jobs and for mechanical difficulties to have been worked out. The system will operate continuously 24 hours a day for 7 days a week. Let $N_{i}$ be the number of 
parts manufactured in the $i$ th hour. If the stochastic process $N_{1}, N_{2}, \ldots$ has a steady-state distribution with corresponding random variable $N$, then we are interested in estimating the steady-state mean $v=E(N)$.

\section{STATISTICAL ANALYSIS FOR TERMINATING SIMULATIONS}

Suppose that we make $n$ independent replications of a terminating simulation each terminated by the event $E$. Let $X_{j}$ be an output random variable defined over the $j$ th replication, for $j=1,2, \ldots, n$; it is assumed that the $X_{j}{ }^{\prime} s$ are comparable for different replications. Then the $X_{j}{ }^{\prime} s$ are IID random variables. For the bank of Example 4, $X_{j}$ might be the average delay $\sum_{i=1}^{N} D_{i} / N$ over a day from the $j$ th replication, where $N$ (a random variable) is the number of customers served in a day and $D_{i}$ is the delay in queue of the $i$ th arriving customer. For the combat model of Example 5, $X_{j}$ might be the number of red tanks destroyed on the $j$ th replication.

Suppose that we would like to obtain a point estimate and confidence interval for the mean $\mu=E(X)$, where $X$ is a random variable defined on a replication as described above. Make $n$ independent replications of the simulation and let $X_{1}, X_{2}, \cdots, X_{n}$ be the resulting IID random variables. Then, by substituting the $X_{j}$ 's into Expressions (1), (2), and (3), we get that $\bar{X}(n)$ is an unbiased point estimator for $\mu$, and an approximate $100(1-\alpha)$ percent confidence interval for $\mu$ is given by

$$
\bar{X}(n) \pm t_{n-1,1-\alpha / 2} \sqrt{S^{2}(n) / n}
$$

Example 7. A small factory consists of a machine and an inspector, as shown in Figure 1. Unfinished parts arrive to the factory with exponential interarrival times having a mean of 1 minute. Processing times at the machine are uniformly distributed on the interval $[0.65,0.70]$ minute, and subsequent inspection times at the inspector are uniformly distributed on the interval $[0.75,0.80]$. (The assumption of uniformity is for ease of exposition, and is not likely to be valid in a real-world application.) Ninety percent of inspected parts are "good" and leave the system immediately; 10 percent of the parts are "bad" and are sent back to the machine for rework. (Both queues are assumed to be of infinite capacity.) The machine is subject to randomly occurring breakdowns. In particular, a new (or freshly repaired) machine will break down after an exponential amount of calendar time with a mean of 6 hours. Repair times are uniform on the interval $[8,12]$ minutes. If a part is being processed when the machine breaks down, then the machine continues where it left off upon the completion of repair. Assume that the factory is initially empty and idle.

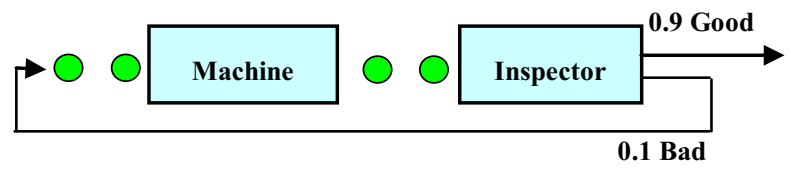

Figure 1: Small Factory

The factory gets an order to produce 2000 parts and, thus, a simulation of this system can be considered to be terminating with $E=\{2000$ parts have been completed $\}$. Let $T$ be the time required to complete the required 2000 parts. Then the company would like a point estimate and a 95 percent confidence interval for the mean $\mu=E(T)$. 
We made 10 independent replications of the simulation and obtained the following observed values for $T$ (in hours):

$$
\begin{gathered}
T_{1}=32.62, T_{2}=32.57, T_{3}=33.51, T_{4}=33.29, \\
T_{5}=32.10, T_{6}=34.24, T_{7}=32.70, T_{8}=33.49, \\
T_{9}=33.36, T_{10}=34.61
\end{gathered}
$$

Substituting the $T_{j}$ 's into Expressions (1), (2), and (3), gives the following results:

$$
\bar{T}(10)=33.25, S^{2}(10)=0.606
$$

and an (approximate) 95 percent confidence interval for $\mu=E(T)$ is given by

$$
33.25 \pm 0.56 \text { or }[32.69,33.81]
$$

Thus, we are approximately 95 percent confident that $\mu$ is between 32.69 and 33.81 hours. (If 100 people performed this experiment independently, then we would expect that about 95 out of the 100 confidence intervals to contain the true $\mu$.) Note also that the interval is quite precise, with the half-length of the confidence interval being less than 2 percent of the point estimate.

\section{STATISTICAL ANALYSIS FOR NONTERMINATING SIMULATIONS}

Let $Y_{1}, Y_{2}, \ldots$ be an output stochastic process from a single run of a non-terminating simulation. Suppose that we want to estimate the steady-state mean $v=E(Y)$, which is also defined by

$$
v=\lim _{i \rightarrow \infty} E\left(Y_{i}\right)
$$

where $E\left(Y_{i}\right)$ is the transient mean at time $i$. Thus, the transient means converge to the steady-state mean. However, $E\left(Y_{i}\right) \neq v$ for "small" $i$ because we generally don't know how to choose the initial conditions $I$ to be representative of "steady-state behavior." This causes the sample mean $\bar{Y}(m)$ to be a biased estimator of $v$ for all finite values of $m$. The problem that we have just described is called the problem of the initial transient in the simulation literature.

The technique most often suggested for dealing with this problem is called warming up the model. The idea is to delete some number of observations from the beginning of a run and to use only the remaining observations to estimate $v$. In particular, given the observations $Y_{1}, Y_{2}, \ldots, Y_{m}$, we would use

$$
\bar{Y}(m, l)=\frac{\sum_{i=l+1}^{m} Y_{i}}{m-l}
$$

$(1 \leq l \leq m-1)$ rather than $\bar{Y}(m)$ as an estimator of $v$. In general, one would expect $\bar{Y}(m, l)$ to be less biased than $\bar{Y}(m)$, since the observations near the "beginning" of the simulation may not be very representative of steady-state behavior due to the choice of initial conditions. 
The question naturally arises as to how to choose the warmup period (or deletion amount) $l$. We would like to pick $l$ (and $m$ ) such that $E[\bar{Y}(m, l)] \approx v$. If $l$ and $m$ are chosen too small, then $E[\bar{Y}(m, l)]$ may be significantly different than $v$. On the other hand, if $l$ is chosen larger than necessary, then $\bar{Y}(m, l)$ will probably have an unnecessarily large variance.

The simplest and most general technique for determining $l$ is a graphical technique due to Welch (1983) [see also Law (2007, pp. 509-516)]. Its specific goal is to determine $l$ such that $E\left(Y_{i}\right) \approx v$ for $i>l$, where $l$ is the warmup period. This is equivalent to determining when the transient-mean curve $E\left(Y_{i}\right)$ "flattens out" at level $v$. In general, it is difficult to determine $l$ from a single replication due to the inherent variability of the process $Y_{1}, Y_{2}, \ldots$. As a result, Welch's procedure is based on making multiple replications of the simulation in a pilot study.

\subsection{The Replication/Deletion Approach}

In this section, we discuss how to construct a point estimate and confidence interval for $v$. Suppose that the warmup period has been determined by Welch's procedure or by using "engineering judgment." Make $n$ independent replications of the output process $Y_{1}, Y_{2}, \ldots$ each of length $m$, where $m$ should be much larger than $l$. (There is no definitive way of picking the run length $m$ here, as there was for terminating simulations.) Let $Y_{j i}$ be the $i$ th observation from the $j$ th replication, for $j=1,2, \ldots, n$ and $i=1,2, \ldots, m$. Let

$$
X_{j}=\sum_{i=l+1}^{m} Y_{j i} /(m-l) \text { for } j=1,2, \ldots, n
$$

Note that $i=l+1$ is where we think that "steady state" begins. Then the $X_{j}$ 's are IID random variables. Furthermore, $E\left(X_{j}\right) \approx v$ since $Y_{j, l+1}, Y_{j, l+2}, \ldots, Y_{j, m}$ each have approximate mean $v$. Then, by substituting the $X_{j}$ 's into Expressions (1), (2), and (3), we get that $\bar{X}(n)$ is an (approximately) unbiased point estimator for $v$, and an approximate $100(1-\alpha)$ percent confidence interval for $v$ is given by

$$
\bar{X}(n) \pm t_{n-1,1-\alpha / 2} \sqrt{S^{2}(n) / n}
$$

We call the above method for constructing a point estimate and confidence interval for $v$ the replication/deletion method. One criticism that has been levied against this method historically is that $l$ observations must be discarded from each of the $n$ replications. However, given the availability and speed of PCs, this is no longer an issue for many, if not most, steady-state analyses.

Example 8. Consider a manufacturing system with a receiving/shipping station and five workstations (see Figure 2), as described in Law (2007, pp. 694-704). Assume that there are 4, 2, 5, 3, and 2 machines in stations 1 through 5, respectively. The machines in a particular station are identical, but machines in different stations are dissimilar. Jobs arrive to the system with exponential interarrival times with a mean of $1 / 15$ of an hour. Thus, 15 jobs arrive in a typical hour. There are three types of jobs, and jobs are of types 1,2 , and 3 , with respective probabilities $0.3,0.5$, and 0.2 . Job types 1,2 , and 3 require 4, 3, and 5 operations to be done, respectively, and each operation must be done at a specified workstation in a prescribed order. Each job begins at the receiving/shipping station, travels to the work stations on its routing, and then leaves the system at the receiving/shipping station. For example, the routing for a type 1 job is $3,1,2,5$. 


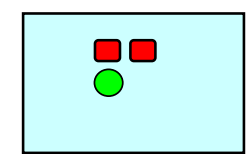

Workstation 2

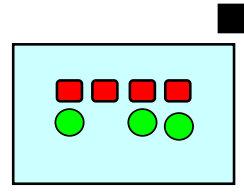

Workstation 1

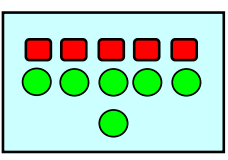

Workstation 3

Forklift

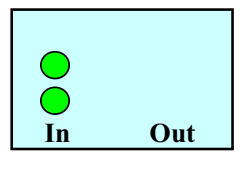

Receiving/Shipping

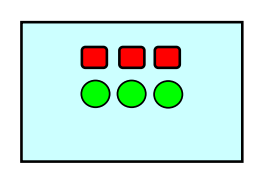

Workstation 4

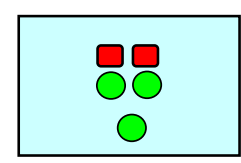

Workstation 5

Figure 2: Factory with five workstations

A job must be moved from one station to another by a forklift truck, which moves at a speed of 5 feet per second. When a forklift becomes available, it processes requests by jobs using a shortestdistance-first dispatching rule. The factory has 2 forklift trucks. Each station has a single FIFO queue. The time to perform an operation at a particular machine is a gamma random variable with a shape parameter of 2, whose mean depends on the job type and the station to which the machine belongs. For example, the mean service time for a type 1 job at station 3 (the first station on its routing) is 0.25 hour. When a machine finishes processing a job, the job blocks that machine (i.e., the machine cannot process another job) until the job is removed by a forklift.

The factory is open 8 hours a day, and thus the arrival rate is 120 jobs per day. The system configuration described here is called system design 3 in Law (2007).

Let $N_{1}, N_{2}, \ldots$ be the output stochastic process corresponding to daily throughputs. Then we are interested in obtaining a point estimate and 90 percent confidence interval for the steady-state mean daily throughput $v=E(N)$. Using Welch's graphical procedure, we determined that a reasonable warmup period for this output process is $l=15$ days [see Law (2007, p. 704)].

We made $n=10$ (production) replications of length $m=115$ days, and used a warmup period of $l=15$ days. Let

$$
X_{j}=\frac{\sum_{i=16}^{115} N_{j i}}{100}
$$

where $N_{j i}$ is the throughput in the $i$ th day of the $j$ th replication.

Substituting the $X_{j}{ }^{\prime} s$ into Expressions (1), (2), and (3), we get the following point estimate and approximate 90 percent confidence interval for $v=E(N)$ :

$$
\hat{v}=\bar{X}(10)=120.29
$$

and

$$
120.29 \pm 0.63 \text { or }[119.66,120.92]
$$

Thus, we are approximately 90 percent confident that the steady-state mean daily throughput is between 119.66 and 120.92 jobs per day. Note that this confidence interval contains 120 , which 
should be the mean daily throughput if the system has enough machines and forklifts. (In a real application, $v$ would not, of course, be known.)

Note also that the confidence interval is quite precise, with the half-length being less than 1 percent of the point estimate.

Also, since $X_{j}$ is the average of $100 \quad N_{j i}{ }^{\prime} s$, it should be approximately normally distributed by a central-limit-theorem type effect. This suggests that the coverage of the confidence interval should be close to the desired coverage probability of 0.9 . Finally, if, for example, we wanted to decrease the half-length by a factor of 3, then a total of approximately 90 replications would be required.

\section{SUMMARY AND PITFALLS IN OUTPUT-DATA ANALYSIS}

We have seen that both terminating and non-terminating analyses can be performed easily by making independent replications of the simulation model and by using Expressions (1), (2), and (3), which come from a first undergraduate course in statistics. In the case of steady-state parameters, we also have to determine a warmup period, but this can be reliably addressed using Welch's graphical approach. The method of replication can also be easily applied to comparing alternative system configurations [see, for example, Law (2007, chapters 10 and 11)] and to estimating multiple measures of performance. Moreover, multiple replications can be made simultaneously on computers having multiple cores or connected by a local-area network.

The following are three major pitfalls in output-data analysis:

- Analyzing simulation output data from one run using formulas [e.g., Expression (2)] that assume independence, which might result in a gross underestimation of variances and standard deviations. This problem is exacerbated by the use of these formulas by some simulation-software packages.

- Failure to have a warmup period for steady-state analyses

- Failure to determine the statistical precision of simulation output statistics by the use of a confidence interval, which can be accomplished easily using the replication approach.

\section{REFERENCES}

Alexopoulos, C. 2007. Statistical analysis of simulation output: state of the art. In Proceedings of the 2007 Winter Simulation Conference, ed. S. G. Henderson, B. Biller, M.-H. Hsieh, J. Shortle, J. D. Tew, and R. R. Barton, 150-161. Piscataway, New Jersey: Institute of Electrical and Electronic Engineers.

Banks, J., J. S. Carson, B. L. Nelson, and D. M. Nicol. 2010. Discrete-event system simulation, $5^{\text {th }}$ ed. Upper Saddle River, New Jersey: Prentice-Hall.

Law, A. M. 2007. Simulation modeling and analysis, 4th ed. New York: McGraw-Hill.

Nakayama, M. K. 2008. Statistical analysis of simulation output. In Proceedings of the $2008 \mathrm{Winter} \mathrm{Si-}$ mulation Conference, ed. S. J. Mason, R. R. Hill, L. Mönch, O. Rose, T. Jefferson, and J. W. Fowler, 62-72. Piscataway, New Jersey: Institute of Electrical and Electronic Engineers.

Welch, P. D. 1983. The statistical analysis of simulation results. In The Computer Performance Modeling Handbook, ed. S.S. Lavenberg. New York: Academic Press. 


\section{AUTHOR BIOGRAPHY}

AVERILL M. LAW is President of Averill M. Law \& Associates, a company specializing in simulation seminars, simulation consulting, and software. He has been a simulation consultant to numerous organizations including Accenture, ARCO, Boeing, Defense Modeling and Simulation Office, Hewlett-Packard, Kimberly-Clark, M\&M Mars, Oak Ridge National Lab, 3M, U.S. Air Force, U.S. Army, and U.S. Navy. He has presented more than 490 simulation short courses in 18 countries. He has written or coauthored numerous papers and books on simulation, operations research, statistics, and manufacturing including the book Simulation Modeling and Analysis that has more than 129,000 copies in print and is widely considered to be the "bible" of the simulation. He developed the ExpertFit ${ }^{\circledR}$ distribution-fitting software and also several videotapes on simulation modeling. He won the INFORMS Simulation Society Lifetime Professional Achievement Award for 2009. He has been the keynote speaker at simulation conferences worldwide. He wrote a regular column on simulation for Industrial Engineering magazine. He has been a tenured faculty member at the University of Wisconsin-Madison and the University of Arizona. He has a Ph.D. in industrial engineering and operations research from the University of California at Berkeley. His e-mail address is <averillesimulation.ws> and his website is <www.averilllaw. com>. 